\title{
KPHMMER: Hidden Markov Model generator for detecting KEGG PATHWAY-specific genes
}

\author{
Hirotaka Suetake ${ }^{1}$ and Masaaki Kotera ${ }^{2 *}$ \\ 1 Department of Creative Informatics, Graduate School of Information Science and Technology, The \\ University of Tokyo, 7-3-1 Hongo, Bunkyo-ku, Tokyo, 113-8656, JAPAN.
}

2 Department of Chemical System Engineering, School of Engineering, The University of Tokyo, 7-31 Hongo, Bunkyo-ku, Tokyo, 113-8656, JAPAN.

*To whom correspondence should be addressed.

Associate Editor: $X X X X X X X$

\section{Received on XXXXX; revised on XXXXX; accepted on XXXXX}

\begin{abstract}
Motivation: Reinforcement of HMMER search for secondary metabolism-specific Pfam domains should contribute to discover novel biosynthetic machinery of clinically important natural products.

Results: Here we provide a Python-based command line tool, named as KPHMMER, to extract the Pfam domains that are specific in the user-defined set of pathways in the user-defined set of organisms registered in the KEGG database. KPHMMER outperformed the previous study in detecting secondary metabolism-specific Pfam domain set. Furthermore, it was proven that KPHMMER helps reduce the computational cost compared with the case using the whole Pfam-A HMM file. We believe that KPHMMER is a powerful tool enabling to deal with many other genome-sequenced species for more general purpose.
\end{abstract}

Availability: KPHMMER is implemented as a Python package freely available via the package management system "pip" and also at https://github.com/suecharo/KPHMMER

Contact: maskot@chemsys.t.u-tokyo.ac.jp

\section{Introduction}

Metabolic pathways are classified into two categories: i.e., those that include ubiquitous chemical compounds such as nucleic acids, proteins and sugars, which are essential for the survival of the living organisms, and those that include species- or clade-specific compounds for the use of interspecies interaction or environmental adaptation, such as toxins or pigments. Even though, the distinction between primary and secondary metabolism is vague (Kotera et al., 2008). Some secondary metabolites are important sources of antibiotics, anticancer drugs, etc. The genome sequences of various organisms revealed that some actinomycetes have genetic potential to synthesize more secondary metabolites than those known before the genome sequencing (Ohmura et al., 2001). This finding indicates that more detailed and comprehensive annotation of secondary metabolism-specific gene sets should allow us to discover novel biosynthetic machinery of clinically important natural products.
Pfam database (Finn et al., 2015) contains so-called Pfam domains, which are protein domains conserved structurally and functionally. These Pfam domains are used as clues to annotate functions of proteins or genes. HMMER (Prakash et al., 2017) is a widely used tool to search for those domains, and is useful especially for putative proteins that cannot be annotated by BLAST-based homology search (Altschul et al., 1997).

The 2ndFind webserver http://biosyn.nih.go.jp/2ndfind/ predicts protein-coding genes using MetaGeneAnnotator (Noguchi et al., 2008) or AUGUSTUS (Stanke and Burkhard, 2005), and the obtained putative genes are subjected to HMMER against the Pfam domains of secondary metabolism. In this previous work, 2ndFind prepared gene sets from the genome sequences of four actinomycetes species, and the obtained genes were categorized into primary or secondary metabolism. Pfam domains were categorized into those found in predominantly in primary or secondary metabolism: if a domain was found more frequently in secondary metabolism, then the domain was regarded to be secondary-specific. 
H.Suetake et al.

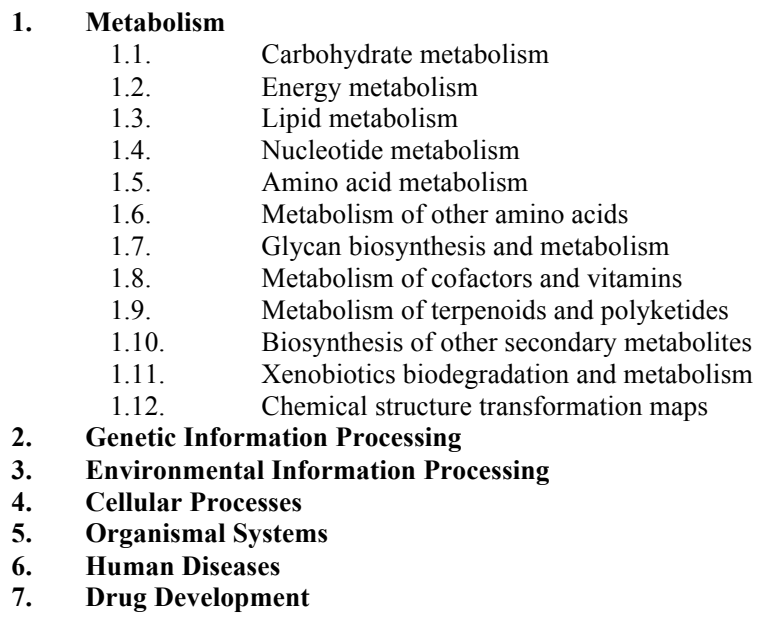

Scheme 1. Hierarchy of KEGG PATHWAY

The previous work is valuable for the study of Streptomyces and related species, although there still remain rooms to improve. First, the secondary-specific Pfam domains were determined just by comparing the numbers of occurrences in primary and secondary metabolism, even though the fact that the numbers of genes in primary and secondary metabolisms differ substantially. Secondly, the distinction criterion of primary and secondary metabolism was not clear.

Here we provide a Python-based command line tool, named as KPHMMER, to extract the Pfam domains that are specific in the userdefined set of pathways (e.g., secondary metabolism) from others (e.g., primary metabolism) in the user-defined set of organisms registered in the KEGG PATHWAY database (Kanehisa et al., 2016). We show in this paper that KPHMMER outperformed the previous study in detecting secondary-specific Pfam domain set. Furthermore, it was proven that KPHMMER helps reduce the computational cost to extract genes containing the user-defined specific Pfam domain sets in the user-defined set of organisms, compared with the case using the whole Pfam-A HMM file. We believe that KPHMMER is not limited to the study of secondary metabolism, but is a powerful tool enabling to deal with many other genome-sequenced species for more general purpose.

\section{Methods}

\subsection{Definition of primary and secondary metabolism}

We used KEGG PATHWAY database, which classifies pathways into seven classes (Scheme 1). Among them, the first class (1. metabolism) further classifies the pathways into 12 subclasses. In this study, subclasses 1.9 (Metabolism of terpenoids and polyketides), 1.10 (Biosynthesis of other secondary metabolites) and 1.12 (Chemical structure transformation maps) are regarded as secondary, and the remaining subclasses were regarded as primary. We designed KPHMMER software so that the user can reuse this method to classify pathways into two with the user's own definitions.

\subsection{Protein-coding gene sets of six actinomycetes species}

The protein-coding gene sets were also obtained from KEGG database, and we designed KPHMMER so that the users can reuse this method for

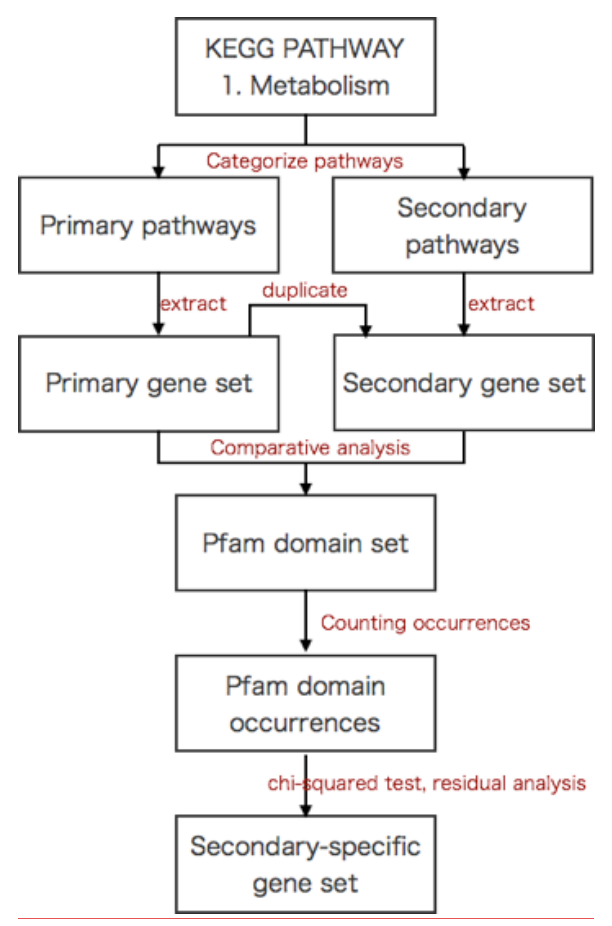

Figure 1. Proposed KPHMMER procedure

any KEGG-registered organisms of the user's interest. In this study, for the performance comparison with the previous study, gene sets were retrieved from six actinomycetes species (Streptomyces avermitilis, Streptomyces coelicolor, Streptomyces griseus, Saccharopolyspora erythraea, Streptomyces scabiei and Streptomyces venezuerae). w

\subsection{Secondary metabolism-specific Pfam domains and genes}

Figure 1 illustrates the proposed procedure to obtain secondaryspecific Pfam domains and genes. After categorizing pathways into primary and secondary, the genes in the selected species were classified into primary and secondary gene sets according to the pathways to which the genes belong.

In the case where the genes dually appear in primary and secondary pathways, they were classified into secondary ("duplicate" in Figure 1) based on the fact that some "primary" metabolites are used as the intermediate compounds to synthesize secondary metabolites, and some even act as inter-species interaction or environmental adaptation like secondary metabolites. KPHMMER has an option for the user to select which category the user would like to put these dually appeared genes into.

The numbers of occurrences of the genes in primary and secondary gene sets were counted for each Pfam domain. We regarded the Pfam domains and genes as secondary-specific when the occurrence of the Pfam domains was significantly greater in secondary metabolism based on the chi-squared test.

\section{Results}

\subsection{Implementation of KPHMMER}


bioRxiv preprint doi: https://doi.org/10.1101/636290; this version posted May 14, 2019. The copyright holder for this preprint (which was not certified by peer review) is the author/funder. All rights reserved. No reuse allowed without permission.

HMM generator for KEGG PATHWAY-specific genes

We implemented a Python-based command line tool named KPHMMER. This tool accepts any genome-sequenced organisms registered in KEGG as the input queries, and outputs the gene set, protein set and Pfam domain set belonging to the category of the user's interest (e.g., the sets significantly found in secondary metabolism). We optimized the API query in this tool to reduce the number of GET request to the KEGG REST API server as many as about 100. This tool also retrieves unstructured KEGG data.

KPHMMER consists of five submethods:

- search receives a keyword and search for KEGG organism code (e.g., hsa for Homo sapiens).

- query receives the KEGG organism code, and outputs the Pfam domains as a yaml file.

- config checks or changes the configuration, i.e., the two categories of the pathways to compare, and also the user's decision which categories the dually appeared genes should be classified into.

- analysis receives Pfam domain yaml file, and outputs a tsv file listing Pfam domains or HMM that frequently observed only in the selected category.

- convert receives Pfam domain yaml file, and outputs the gene contained therein as fasta file.

An advantage of this tool is its versatility: the user can input any combinations of genome-sequenced organisms (not only the actinomycetes species used in this work), can choose any combinations of pathways of the user's interest (not only for the distinction of metabolic pathways as shown here, but also of any pathways as listed in Scheme 1), and can obtain Pfam domains or amino acid sequences that specifically occur in the selected set of pathways.

\subsection{Performance comparison on extracting the secondary- specific domains and genes}

According to the previous study, 2ndFind detected 3039 Pfam domains from the 29,728 protein-coding genes found in four actinomycetes species (Streptomyces avermitilis ATCC 31267, Streptomyces coelicolor A3(2), Streptomyces griseus NBRC 13350, Saccharopolyspora erythraea NRRL 2338), of which 82 were regarded as secondary-specific Pfam domains (as listed in the webpage http://biosyn.nih.go.jp/2ndfind/), of which three domains (HxxPF_rpt, Lant_dehyd_N, and NRPS) were no longer alive entries but were merged into Condensation (PF00668), Lant_dehydr_N (PF04738), and Condensation (PF00668), respectively. As the result, the number of the current version of secondary-specific Pfam domains was 81 .

Table 1. Performance comparison

\begin{tabular}{|c|c|c|c|c|c|c|}
\hline & $\mathrm{TP}$ & FP & $\mathrm{FN}$ & $\mathrm{TN}$ & Precision & Recall \\
\hline \multicolumn{7}{|c|}{ Streptomyces scabiei } \\
\hline 2ndFind & 36 & 122 & 71 & 892 & 0.227 & 0.336 \\
\hline KPHMMER & 100 & 200 & 7 & 814 & 0.333 & 0.934 \\
\hline \multicolumn{7}{|c|}{ Streptomyces venezuerae } \\
\hline 2ndFind & 46 & 114 & 74 & 830 & 0.287 & 0.383 \\
\hline KPHMMER & 113 & 180 & 7 & 764 & 0.385 & 0.941 \\
\hline \multicolumn{7}{|c|}{$\begin{array}{l}\text { TP (True Positive): the number of genes that belong to secondary metabolism, } \\
\text { and were correctly predicted as secondary metabolism. FP (False Positive): the } \\
\text { number of genes that belong to primary metabolism, but were falsely predicted as } \\
\text { secondary metabolism. FN (False Negative): the number of genes that belong to } \\
\text { secondary metabolism, but were falsely predicted as primary metabolism. TN } \\
\text { (True Negative): the number of genes that belong to primary metabolism, and } \\
\text { were correctly predicted as primary metabolism. Precision: TP / (TP + FP). Re- } \\
\text { call: TP / (TP + FN). }\end{array}$} \\
\hline
\end{tabular}

On the contrary, using the same four actinomycetes species, KPHMMER detected 2,036 Pfam domains from 4,410 metabolismrelated genes, of which 28 were regarded as secondary-specific. It was found that the overwrap of the secondary-specific Pfam domains was only five (Aminotran_1_2, KR, NAD_binding_9, ketoacyl-synt, Thiolase_N).

Table 2. Top 40 Pfam domains that were regarded to be secondary-specific by KPHMMER

\begin{tabular}{|c|c|c|c|c|}
\hline Pfam & primary & secondary & $\mathrm{p}$ value & 2ndFind \\
\hline ketoacyl-synt & 18 & 53 & $6.41 \mathrm{e}-55$ & + \\
\hline Thiolase_C & 0 & 36 & $2.19 \mathrm{e}-54$ & - \\
\hline Thiolase_N & 30 & 54 & $1.74 \mathrm{e}-45$ & + \\
\hline polyprenyl_synt & 0 & 24 & $3.50 \mathrm{e}-36$ & + \\
\hline PP-binding & 1 & 18 & $2.30 \mathrm{e}-25$ & + \\
\hline DHDPS & 6 & 22 & $1.86 \mathrm{e}-24$ & - \\
\hline ROK & 4 & 18 & $4.09 \mathrm{e}-21$ & - \\
\hline GFO_IDH_MocA_C & 0 & 14 & $4.74 \mathrm{e}-21$ & - \\
\hline GDP_Man_Dehyd & 32 & 33 & $1.01 \mathrm{e}-19$ & - \\
\hline SQS_PSY & 0 & 12 & $5.04 \mathrm{e}-18$ & + \\
\hline Epimerase & 66 & 44 & $1.22 \mathrm{e}-17$ & - \\
\hline KAsynt_C_assoc & 1 & 12 & $2.25 \mathrm{e}-16$ & - \\
\hline ECH_2 & 11 & 19 & $6.85 \mathrm{e}-16$ & - \\
\hline ECH_1 & 12 & 19 & $3.43 \mathrm{e}-15$ & - \\
\hline $\mathrm{MbtH}$ & 2 & 12 & $5.81 \mathrm{e}-15$ & + \\
\hline RmlD_sub_bind & 35 & 28 & $1.16 \mathrm{e}-13$ & - \\
\hline Terpene_synth_C & 0 & 9 & $1.76 \mathrm{e}-13$ & + \\
\hline Prenyltransf & 0 & 9 & $1.76 \mathrm{e}-13$ & - \\
\hline NAD_binding_4 & 23 & 22 & $1.30 \mathrm{e}-12$ & - \\
\hline Polysacc_synt_2 & 28 & 24 & $1.56 \mathrm{e}-12$ & - \\
\hline $\mathrm{p} 450$ & 13 & 17 & $3.09 \mathrm{e}-12$ & + \\
\hline NAD_binding_5 & 0 & 8 & $5.80 \mathrm{e}-12$ & - \\
\hline Inos-1-P_synth & 0 & 8 & $5.80 \mathrm{e}-12$ & - \\
\hline Glucokinase & 0 & 8 & $5.80 \mathrm{e}-12$ & - \\
\hline DXP_reductoisom & 0 & 8 & $5.80 \mathrm{e}-12$ & - \\
\hline PS-DH & 0 & 8 & $5.80 \mathrm{e}-12$ & - \\
\hline adh_short & 71 & 38 & $6.01 \mathrm{e}-12$ & - \\
\hline Acyl_transf_1 & 5 & 12 & $9.78 \mathrm{e}-12$ & + \\
\hline 3Beta_HSD & 31 & 24 & $1.81 \mathrm{e}-11$ & - \\
\hline Semialdhyde_dh & 15 & 17 & $3.63 \mathrm{e}-11$ & - \\
\hline Ketoacyl-synt_C & 16 & 17 & $1.11 \mathrm{e}-10$ & + \\
\hline MCRA & 6 & 11 & $1.19 \mathrm{e}-09$ & - \\
\hline Beta-lactamase & 1 & 7 & $5.80 \mathrm{e}-09$ & - \\
\hline DEDD_Tnp_IS110 & 0 & 6 & $6.34 \mathrm{e}-09$ & - \\
\hline Penicil_amidase & 0 & 6 & $6.34 \mathrm{e}-09$ & - \\
\hline GcpE & 0 & 6 & $6.34 \mathrm{e}-09$ & - \\
\hline AXE1 & 0 & 6 & $6.34 \mathrm{e}-09$ & - \\
\hline dTDP_sugar_isom & 0 & 6 & $6.34 \mathrm{e}-09$ & + \\
\hline ACP_syn_III & 23 & 18 & 7.26e-09 & - \\
\hline GFO_IDH_MocA & 18 & 16 & $7.74 \mathrm{e}-09$ & - \\
\hline
\end{tabular}


bioRxiv preprint doi: https://doi.org/10.1101/636290; this version posted May 14, 2019. The copyright holder for this preprint (which was not certified by peer review) is the author/funder. All rights reserved. No reuse allowed without permission.

H.Suetake et al.

Table 3. Comparison of the computational time

\begin{tabular}{lrrr}
\hline & hmmpress & \multicolumn{2}{c}{ hmmscan } \\
& & S. scabiei & S. venezuerae \\
\hline Pfam-A.hmm & $40.6 \mathrm{~s}$ & $10 \mathrm{~m} 31 \mathrm{~s}$ & $10 \mathrm{~m} 58 \mathrm{~s}$ \\
KPHMMER.hmm & $4.6 \mathrm{~s}$ & $1 \mathrm{~m} 57 \mathrm{~s}$ & $1 \mathrm{~m} 44 \mathrm{~s}$ \\
\hline
\end{tabular}

In order to compare the predictive performance of 2 ndFind and KPHMMER, we used two taxonomically close Streptomyces species, i.e., S. scabiei 87.22 (containing 2,036 Pfam domains in 1,021 metabolismrelated genes) and S. venezuerae ATCC 10712 (containing 2,036 Pfam domains in 1,068 metabolism-related genes). Both of these Streptomyces species are registered in KEGG, and the genes were annotated in either primary or secondary metabolism. This annotation was regarded as the correct data for the performance comparison.

In this study, we used the chi-squared test (p-value $<0.05$ ) to extract secondary-specific Pfam domains in KPHMMER. Table 1 shows the confusion matrices. Performance was evaluated by precision and recall metrics using the numbers of genes classified in primary and secondary metabolisms. Our KPHMMER method outperformed the previous study in both terms of precision and recall in both species. Especially, recall improved dramatically by using KPHMMER. Table 2 shows the top 40 Pfam domains that were regarded as secondary-specific using KPHMMER. This result demonstrates that KPHMMER successfully retrieves some Pfam domains that are known to appear frequently in secondary metabolism but are not listed in 2ndFind (such as KAsynt_C_assoc, and Prenyltransf).

\subsection{Reduction of computational cost by using KPHMMER}

Here we further present the effectiveness of our KPHMMER strategy on the reduction of computational costs. Pfam-A.hmm file contains all families of domains defined in Pfam. Pfam 31.0 (as of March 2017, ftp://ftp.ebi.ac.uk/pub/databases/Pfam) contains 16712 entries, and the size of this file is about $1.3 \mathrm{~Gb}$. KPHMMER helps the user reduce the numbers of Pfam domains as the user's need. For example, when we selected the four actinomycetes species (Streptomyces avermitilis, Streptomyces coelicolor, Streptomyces griseus, Saccharopolyspora erythraea) in this study, the obtained HMM file (named as KPHMMER.hmm) only contained 2,036 domains and had $171 \mathrm{Mb}$.

Table 3 shows the comparison of the computational time to conduct Pfam search. The fasta file used in this study was not very big $(\sim 500 \mathrm{~Kb})$, and the hmmscan using Pfam-A.hmm took about 11 minutes, compared with about 2 minutes using KPHMMER.hmm. However, this difference should be more serious when the users deal with larger fasta files.

\section{Summary}

We established a Python-based generic command line tool named KPHMMER, by which the user can generate Pfam domain sets found significantly in the set of pathways of user's interest, from the organisms of the user's interest. KPHMMER accepts not only for Streptomyces species but also any genome-sequenced species registered in KEGG. The obtained Pfam domain sets should be valuable to the annotation of the genes derived form the closely related species. As the future work, KPHMMER has potential for the enrichment analysis combined with Gene Ontology for transcriptome data, as well as discovering the gene clusters in the genome sequences that contribute to valuable natural product biosynthesis.

\section{Funding}

Japan Society for the Promotion of Science (JSPS) Kakenhi [17K07260].

Conflict of Interest: none declared.

\section{References}

Altschul, Stephen F., et al. "Gapped BLAST and PSI-BLAST: a new generation of protein database search programs." Nucleic acids research 25.17 (1997): 33893402 .

Finn, Robert D., et al. "The Pfam protein families database: towards a more sustainable future." Nucleic acids research 44.D1 (2015): D279-D285.

Kanehisa, Minoru, et al. "KEGG: new perspectives on genomes, pathways, diseases and drugs." Nucleic acids research 45.D1 (2016): D353-D361.

Kotera M, et al. Eliciting possible reaction equations and metabolic pathways involving orphan metabolites." J Chem Inf Model . 2008 Dec;48(12):2335-49.

Noguchi, Hideki, Takeaki Taniguchi, and Takehiko Itoh. "MetaGeneAnnotator: detecting species-specific patterns of ribosomal binding site for precise gene prediction in anonymous prokaryotic and phage genomes." DNA research 15.6 (2008): 387-396.

Omura, Satoshi, et al. "Genome sequence of an industrial microorganism Streptomyces avermitilis: deducing the ability of producing secondary metabolites." Proceedings of the National Academy of Sciences of the United States of America 98.21 (2001): 12215-12220.

Prakash, Ananth, et al. "The HMMER Web Server for Protein Sequence Similarity Search." Current protocols in bioinformatics (2017): 3-15.

Stanke, Mario, and Burkhard Morgenstern. "AUGUSTUS: a web server for gene prediction in eukaryotes that allows user-defined constraints." Nucleic acids research 33.suppl_2 (2005): W465-W467. 